\title{
Influencia de la atmósfera de sinterización en las propiedades mecánicas de los aceros P/M AISI 430L
}

\author{
F. Javier Iglesias ${ }^{\mathrm{a}, \bigotimes}$, Carmen Martínez $^{\mathrm{a}}, \mathrm{M}^{\mathrm{a}}$ Teresa Cotes $^{\mathrm{a}}$, \\ José Manuel Ruiz $^{\mathrm{b}}$, Luis E. García ${ }^{\mathrm{b}}$, Francisco Corpas ${ }^{\mathrm{a}}$ \\ ${ }^{a}$ E.P.S. Linares, Universidad de Jaén, C/ Alfonso X el Sabio, 28. 23700 Linares (Jaén), España \\ ${ }^{\text {b}}$ E.T.S. de Ingenieros de Minas, Universidad Politécnica de Madrid, C/ Ríos Rosas, 21, 28003 Madrid, España \\ $\triangle$ Autor para la correspondencia: figodino@ujaen.es
}

Enviado: 3 Abril 2013; Aceptado: 20 Octubre 2013

\begin{abstract}
RESUMEN: Se ha estudiado el acero inoxidable pulvimetalúrgico AISI 430L, comparando la sinterización en dos atmósferas diferentes; en vacío, y en una atmósfera que contiene nitrógeno. Se ha desarrollado un tratamiento térmico con objeto de incrementar las propiedades mecánicas, mediante la modificación microestructural de los nitruros complejos de hierro y cromo precipitados durante la etapa de sinterización. Se han evaluado las propiedades físicas y a la vez se ha realizado un análisis microestructural con el fin de relacionar la microestructura con el incremento en las propiedades mecánicas.
\end{abstract}

PALABRAS CLAVE: Acero inoxidable AISI 430L; Corrosión; Nitrógeno; Propiedades mecánicas; Sinterización

Citation / Cómo citar este artículo: Iglesias, F.J., Martínez, C., Cotes, M.T., Ruiz, J.M., García, L.E., Corpas, F.A. (2014) "Influencia de la atmosfera de sinterización en las propiedades mecánicas de los aceros P/M AISI 430L" Rev. Metal. 50(1): e008. doi: http://dx.doi.org/10.3989/revmetalm.008

SUMMARY: Influence of sintering atmosphere on the mechanical properties of steel P / M AISI 430L. It has studied the stainless steel powder metallurgy AISI 430L. It has compared the sintering in two different atmospheres; in vacuum, and in an atmosphere containing nitrogen. It has developed a heat treatment with the aim of improving the mechanical properties. This has been done through microstructural modification of complex nitrides of iron and chromium precipitates during the phase of sintering. Physical properties have been evaluated and are been performing a microstructural analysis for microstructure related to the increase in mechanical properties.

KEYWORDS: AISI 430L stainless steel; Corrosion; Mechanical properties; Nitrogen; Sintering

Copyright: (C) 2014 CSIC. This is an open-access article distributed under the terms of the Creative Commons Attribution-Non Commercial (by-nc) Spain 3.0 License.

\section{INTRODUCCIÓN}

La importancia de la industria pulvimetalúrgica en las últimas décadas es atribuible al ahorro de costes asociados a la eliminación de procesos de acabado. Asimismo, cabe destacar que el desarrollo de nuevos materiales y tecnologías de fabricación ha permitido incrementar el número de aplicaciones para estos materiales obtenidos mediante un proceso pulvimetalúrgico.

Los campos de aplicación de las piezas obtenidas por metalurgia de polvos son diversos, ya que ofrecen ventajas como disminución en los costes de fabricación por eliminación de procesos de acabado, 
bajas temperaturas de procesado, y posibilidad de controlar las microestructuras obtenidas (Capus, 2000; Schaefer y Trombino, 2005).

El desarrollo tecnológico de este tipo de materiales ha buscado la mejora de las propiedades mecánicas a través de la optimización de las aleaciones y de los procesos de producción, así como con el desarrollo de nuevas técnicas de aleación para crear materiales con propiedades específicas (Endo, 2002). La presencia inherente de porosidad interconectada o aislada en los aceros inoxidables pulvimetalúrgicos influye de manera importante sobre sus propiedades mecánicas y sobre sus propiedades químicas finales (Molins et al., 1992; Blanco et al., 2005; García et al., 2009). Por otro lado, para los aceros inoxidables pulvimetalúrgicos, los parámetros del proceso productivo marcan las características finales del producto (Corpas et al., 2005).

El hecho de utilizar partículas de tan diferente tamaño y morfología va a condicionar todo el proceso. La elección correcta del proceso de sinterización es muy importante, hay que utilizar las condiciones de temperatura, tiempo y atmósfera adecuadas para obtener la mejor calidad final de las piezas. El mejor valor de la densidad final dependerá de una correcta sinterización y vendrá condicionado por una elevada densidad en verde, generando una microestructura adecuada en términos de porosidad (García et al., 2009).

La atmósfera de sinterización debe cumplir una serie de funciones, como proteger el material de la oxidación durante el proceso de sinterización, reducir los óxidos superficiales a fin de mejorar el contacto entre las partículas adyacentes, impedir la carburación o descarburación de los compactos fabricados, eliminar residuos de lubricante e impedir reacciones indeseables con la atmósfera (Durdaller, 1997). Son numerosas las investigaciones realizadas en los últimos años, en este sentido, centrándose sobre todo en los aceros inoxidables dúplex (Múnez et al., 2008). Los aceros inoxidables pulvimetalúrgicos debido a su elevada porosidad interconectada o aislada, presentan una mayor área superficial que los productos convencionales, por lo cual, estos son susceptibles de reaccionar con la atmósfera del horno de sinterización, dando compuestos que producen una disminución en la resistencia a corrosión de este tipo de aceros (Dobrzanski et al., 2005).

Los estudios de las diferentes atmósferas de sinterización muestran las mejoras en las propiedades mecánicas de los sinterizados en presencia de nitrógeno (Mariappan et al., 2009). El nitrógeno presente en los aceros inoxidables puede existir en forma de moléculas de gas, de solución sólida intersticial y de nitruros precipitados. Tanto la presencia de moléculas de nitrógeno en los poros como la precipitación de nitruros tienen efectos nocivos en los aceros, mientras que el nitrógeno en solución sólida intersticial provoca mejoras en la resistencia a la corrosión y en las propiedades mecánicas de los aceros inoxidables (Sathiranchinda et al., 2010; Martin et al., 2011).

El nitrógeno presente en la atmósfera de sinterización, difunde rápidamente a las elevadas temperaturas a la que se encuentran los compactos y si la velocidad de enfriamiento no es lo suficientemente rápida, se produce la precipitación de nitruros de cromo en los granos de ferrita, produciéndose un empeoramiento en la resistencia a la corrosión, lo que podría solucionarse mediante enfriamientos rápidos, obteniendo mejoras en las propiedades mecánicas y de resistencia a corrosión (Dobrzanski et al., 2007; García et al., 2010).

Los aceros inoxidables ferríticos, debido a su menor precio, comportamiento frente a la corrosión, propiedades magnéticas, etc., los hace susceptibles de ser aplicados en la fabricación de componentes expuestos a altas temperaturas (Samal y Terrel, 2001; Bautista et al., 2008).

En este trabajo nos hemos centrado en el estudio de los aceros inoxidables ferríticos AISI 430L, debido a una potencial aplicación final, intentando conseguir un material con adecuadas propiedades mecánicas, a la vez que resistente a corrosión a elevadas temperaturas. Se ha realizado un estudio de las propiedades físicas, mecánicas, así como de la microestructura de las distintas series a las que hemos sometido a tratamientos térmicos. Con ello tratamos de modificar la estructura cristalina y la cantidad de precipitados obtenidos durante la sinterización, de forma que se consiga aprovechar la parte positiva de la adsorción de nitrógeno, como es un incremento de las propiedades mecánicas sin perjudicar la resistencia a corrosión. El análisis microestructural nos permite relacionar la microestructura de las distintas series, con las propiedades evaluada.

\section{MATERIALES Y MÉTODOS}

Los materiales objeto de este trabajo de investigación han sido conformados, siguiendo un proceso tradicional pulvimetalúrgico, a partir de polvo de acero inoxidable pre-aleado AISI 430L $(0,018 \% \mathrm{C}$, $1,16 \% \mathrm{Si}, 0,18 \% \mathrm{Mn}, 0,10 \% \mathrm{Ni}, 16,9 \% \mathrm{Cr}$ ), obtenido mediante atomización por agua, por la empresa Coldstream S.A. Estos polvos poseen morfología irregular y distribución de tamaños variable como se puede ver en la micrografía (Fig. 1).

En primer lugar se llevó a cabo la compactación de las piezas y se les sometió al proceso de sinterización, parte en atmósfera de vacío y parte en atmósfera de nitrógeno e hidrogeno. Tras la sinterización y con objeto de modificar la morfología de los nitruros complejos, se procede a la realización de un tratamiento térmico.

La compactación de los polvos se ha realizado en una prensa de 150 toneladas. Todas las probetas 


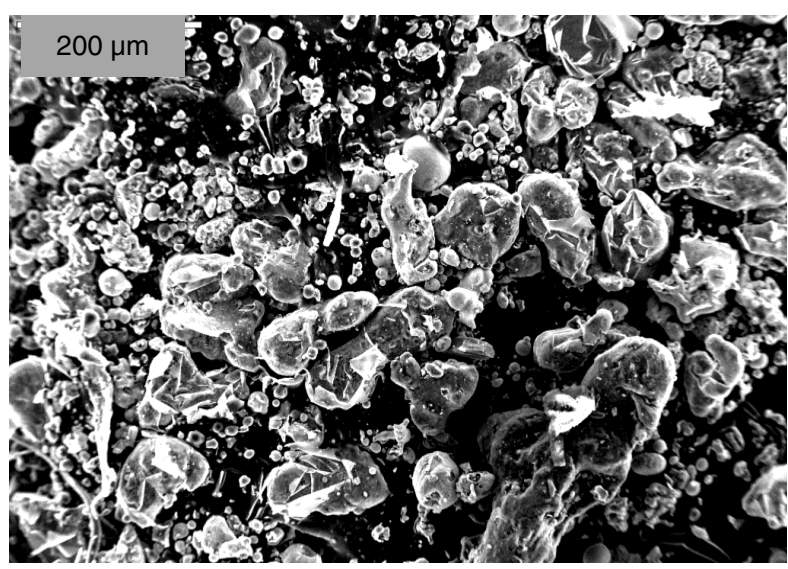

Figura 1. Micrografía de los polvos AISI 430L a 50 aumentos.

se han compactado a $700 \mathrm{MPa}$, empleando matrices flotantes normalizadas, MPIF Standard 10, siguiendo la norma ASTM E-69. En este trabajo se ha empleado lubricante, solamente en las paredes de la matriz, ya que lo que se busca es elevar al máximo la densidad del material, que presenta una mayor área superficial que los aceros fabricados por métodos convencionales, siendo susceptibles de reaccionar con la atmósfera del horno de sinterización. El lubricante utilizado ha sido estearato de zinc.

Para evaluar la temperatura de sinterización de los aceros utilizados hay que buscar un compromiso entre las propiedades finales y la energía mínima necesaria para su procesado. Para ello se realiza un estudio dilatométrico con el fin de evaluar los cambios dimensionales, expansiones y contracciones, que sufre el material por efecto de la temperatura y de las reacciones que ocurren en su interior, que generalmente llevan asociado un cambio de volumen del mismo. El estudio se realizó con un dilatómetro Bahr con atmósfera de vacío, obteniendo un termograma que registra la variación de la longitud en función de la temperatura, observándose en el termograma de la Figura 2, que la temperatura de $1250^{\circ} \mathrm{C}$, es la más apropiada para la sinterización de estos aceros.

Es necesario controlar los parámetros de sinterización, ya que los aceros inoxidables tienen como principal elemento de aleación el cromo y es conocida su gran avidez por el oxígeno. La oxidación de este elemento daría lugar a una pérdida de sus propiedades mecánicas. La sinterización se ha realizado, parte bajo una atmósfera protectora de $95 \% \mathrm{~N}_{2}+5 \% \mathrm{H}_{2}$, utilizando un horno tubular de laboratorio Termiber, con muy bajo contenido en vapor de agua, para evitar la formación de óxidos superficiales. También se ha realizado sinterización bajo atmósfera de vacío, ya que es el procesado normal de este tipo de aceros, para así poder tener una referencia comparativa de los resultados obtenidos y poder comprobar la viabilidad del método seleccionado. Para ello se ha utilizado un horno tubular de laboratorio Carbolite, modelo HVT 15/75, que alcanza una temperatura máxima de $1500{ }^{\circ} \mathrm{C}$, empleando varillas de carburo de silicio como elemento calefactor, aunque la sinterización se ha realizado a $1250^{\circ} \mathrm{C}$.

El ciclo de sinterización, que se ha utilizado para todos los materiales ha sido un calentamiento a $5^{\circ} \mathrm{C} \mathrm{min}^{-1}$, hasta una temperatura de $1250{ }^{\circ} \mathrm{C}$, isoterma a la temperatura de sinterización durante 30 minutos y un enfriamiento posterior a $5{ }^{\circ} \mathrm{C} \mathrm{min}^{-1}$, hasta alcanzar $\operatorname{los} 500{ }^{\circ} \mathrm{C}$, a partir de esa temperatura enfriamiento al aire.

Tras la sinterización, y con el objetivo de modificar la morfología eutectoide de los nitruros complejos de hierro y cromo precipitados, se procede a la realización de un tratamiento de solubilización, hipertemple y maduración. Para el tratamiento de temple se utilizó un horno con cámara Inconel a $950{ }^{\circ} \mathrm{C}$ en atmósfera de argón, con una velocidad de calentamiento de $5^{\circ} \mathrm{C} \mathrm{min}^{-1}$, manteniendo la temperatura 30 minutos y enfriando posteriormente en agua. Para el proceso de maduración se utilizó igualmente

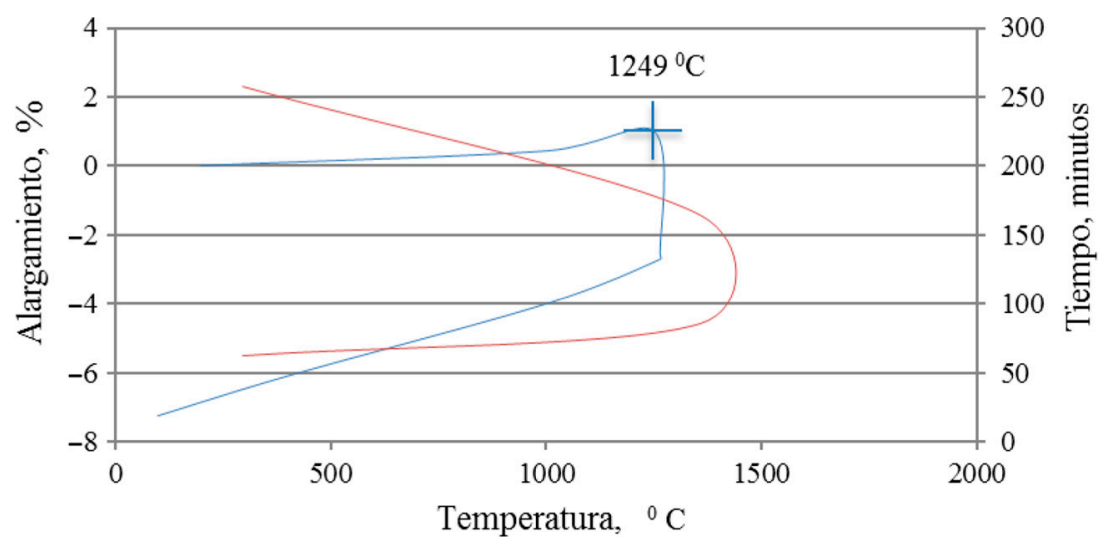

FIGURA 2. Estudio dilatométrico del acero AISI 430L. 
una velocidad de calentamiento de $5{ }^{\circ} \mathrm{C} \min ^{-1}$, en atmósfera inerte de argón, alcanzando temperaturas de maduración desde $100{ }^{\circ} \mathrm{C}$ hasta $700^{\circ} \mathrm{C}$, en intervalos de $100{ }^{\circ} \mathrm{C}$.

A las probetas en verde, después del proceso de compactación, se les ha determinado la densidad, mediante la aplicación de la norma MPIF Standard 42, que consiste en determinar la densidad por medio del principio de Arquímedes.

El estudio de la variación dimensional se obtiene mediante la comparación de las medidas de longitud realizadas antes y después del proceso de sinterización, debidas a la contracción que se ha producido durante la misma. Estos materiales disponen de una gran cantidad de poros, susceptibles de experimentar variaciones dimensionales notables.

La determinación del volumen del material, se realiza mediante la diferencia en peso con y sin inmersión, por lo que al tratarse de materiales porosos, es necesario el sellado con pintura de densidad conocida, previo a la inmersión. Las medidas se realizan en una balanza Salter de precisión $0,0001 \mathrm{~g}$.

La porosidad es una propiedad inherente de los materiales pulvimetalúrgicos, y que afectará en gran medida, al comportamiento mecánico y químico de este tipo de materiales. Se han determinado dos tipos de porosidades:

1. Porosidad total. Este tipo de porosidad engloba tanto la porosidad aislada como la interconectada.

2. Porosidad superficial. Esta porosidad presenta una gran influencia en las propiedades mecánicas del material y una gran disminución de la resistencia a la corrosión de los materiales pulvimetalúrgicos, debido al aumento del área superficial. La determinación de este tipo de porosidad se ha realizado mediante el método de Arquimedes y la distribución, forma y tamaño de los poros mediante el análisis de las micrografías obtenidas mediante microscopía.

La dureza es una propiedad mecánica estrechamente relacionada con las propiedades físicas y microestructurales del material. Se ha utilizado el ensayo de dureza Vickers en la escala HV10 realizando cinco medidas por cada cara de la probeta. Para ello se ha empleado un durómetro Mitutoyo $A V K-A 2$.

Se realizaron ensayos de tracción siguiendo la norma ASTM E8M-00, para la determinación de la resistencia a tracción y el alargamiento que sufren los materiales hasta que rompen. Se han hecho tres ensayos para cada serie, utilizando probetas de geometría tipo dog-bone, con una máquina universal de ensayos Microtest con célula de carga de 20 KN y una velocidad de ensayo de $1 \mathrm{~mm} \mathrm{~min}^{-1}$.

\section{RESULTADOS Y DISCUSIÓN}

Las propiedades de los aceros inoxidables pulvimetalúrgicos pueden aproximarse a aquellos otros obtenidos por técnicas de procesamiento tradicionales. Aunque es bien conocida que la porosidad inherente del proceso pulvimetalúrgico influye de forma negativa en las propiedades mecánicas de los materiales sinterizados, es necesario remarcar que las técnicas de aleación del polvo y la microestructura del material juegan un papel decisivo en el comportamiento de la pieza.

Se han evaluado las propiedades físicas y mecánicas, así como la microestructura de las distintas series que hemos sometido a tratamientos térmicos. Con ello tratamos de modificar la morfología eutectoide de los nitruros complejos de hierro y cromo y la cantidad de precipitados obtenidos durante la sinterización, de forma que se consiga aprovechar la parte positiva de la adsorción de nitrógeno, como es un incremento de las propiedades mecánicas sin perjudicar la resistencia a corrosión.

\subsection{Densidad del sinterizado}

En la Figura 3 se muestra la densidad de los materiales sinterizados en las dos atmósferas utilizadas, respecto a la densidad en verde obtenida, medida por el método de inmersión de Arquímedes.

Para una misma temperatura de sinterización y utilizando el mismo lubricante, se puede observar una mayor densidad de los compactos en atmósfera de vacío, que los mismos sinterizados en atmósfera de $95 \% \mathrm{~N}_{2}-5 \% \mathrm{H}_{2}$, obteniendo valores de densidad de $6,80 \mathrm{~g} \mathrm{~cm}^{-3}$ a $1250{ }^{\circ} \mathrm{C}$.

Para conocer la efectividad del proceso de sinterización, se han calculado los parámetros de densificación en las dos atmósferas, obteniéndose valores un $3,17 \%$ superiores en los sinterizados en vacío que en atmósfera de $95 \% \mathrm{~N}_{2}-5 \% \mathrm{H}_{2}$.

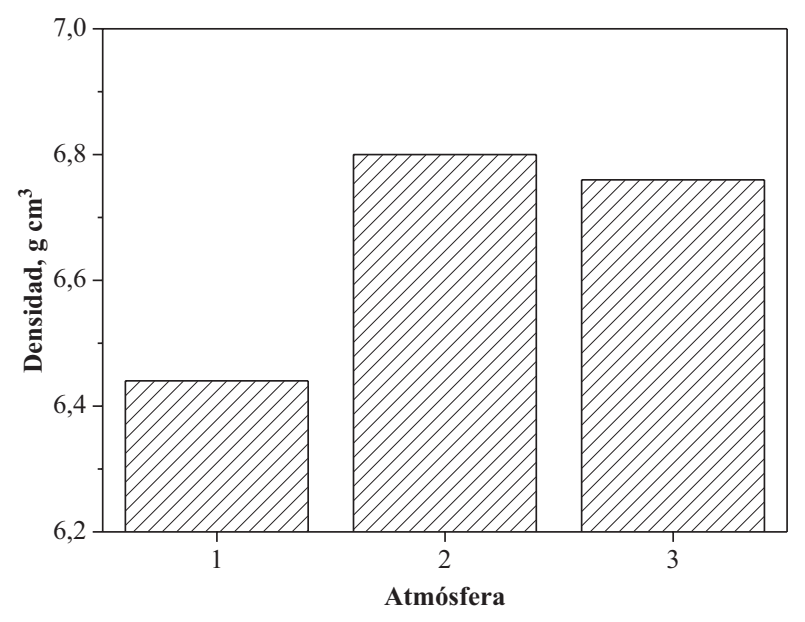

FIGURA 3. Densidad del acero AISI 430L sinterizado en distintas atmósferas: 1) verde; 2) vacío; 3) $95 \% \mathrm{~N}_{2}+5 \% \mathrm{H}_{2}$ 
a)

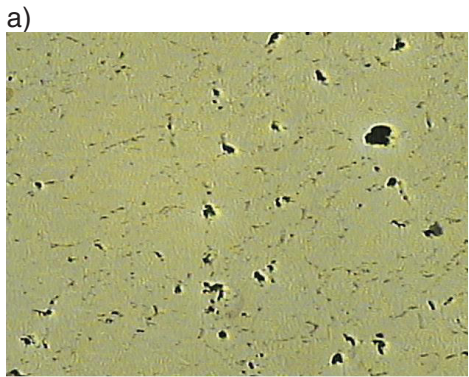

d)

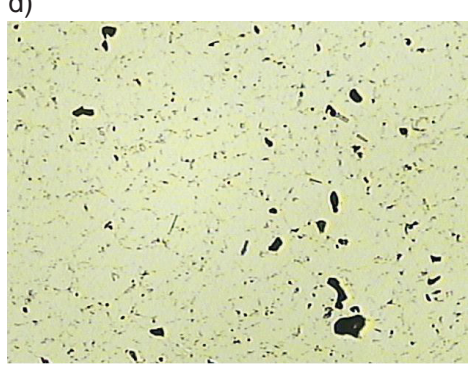

g)

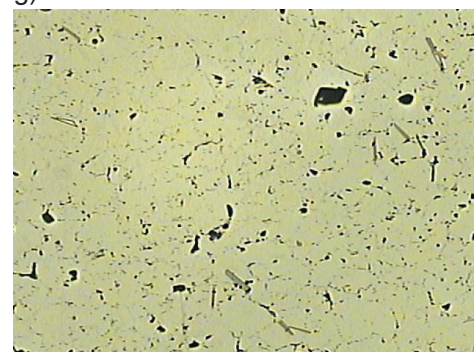

b)

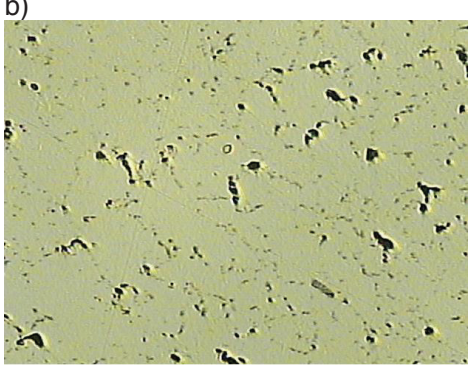

e)

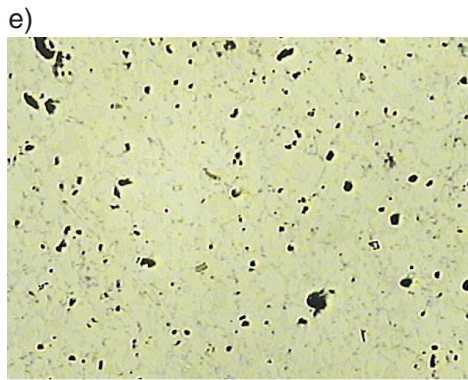

h)

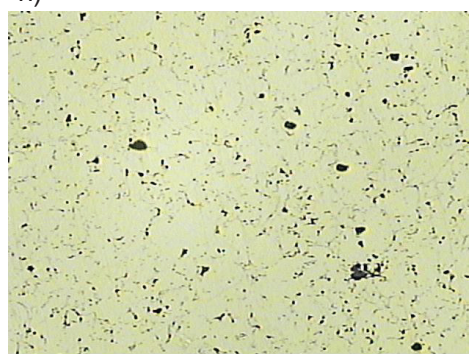

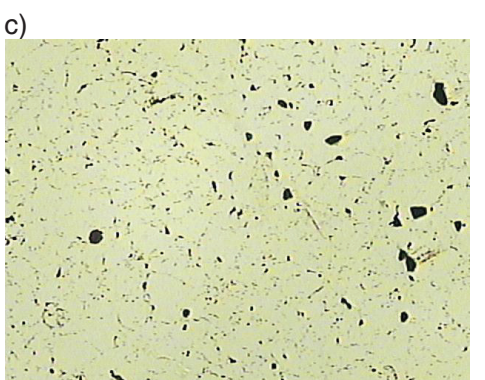

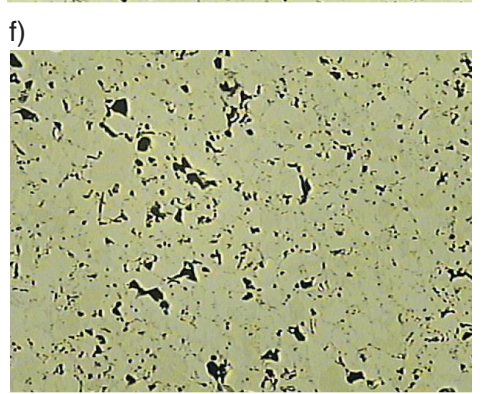

i)

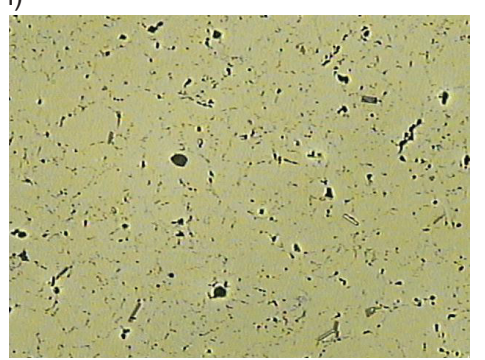

FigurA 4. a) Acero Inoxidable AISI 430L sinterizado en vacío; b) acero inoxidable AISI 430L sinterizado en $\mathrm{N}_{2}-\mathrm{H}_{2}$; c) madurados a $100{ }^{\circ} \mathrm{C}$; d) madurados a $200{ }^{\circ} \mathrm{C}$; e) madurados a $300^{\circ} \mathrm{C}$; f) madurados a $400{ }^{\circ} \mathrm{C}$; g) madurados a $500^{\circ} \mathrm{C}$; h) madurados a $600^{\circ} \mathrm{C}$; i) madurados a $700^{\circ} \mathrm{C}$.

Todo esto es debido a que al sinterizar en presencia de nitrógeno se favorece la formación de nitruros complejos de hierro y cromo que impiden una mayor densificación (Mariappan et al., 2009).

El análisis de la evolución de la porosidad, es fundamental para saber la repercusión que ésta va a tener en el posterior comportamiento del acero frente a la corrosión. Los valores de porosidad total y porosidad superficial, están relacionados con los resultados de densidad, obteniéndose también valores superiores para aquellos aceros sinterizados en presencia de nitrógeno y alcanzando valores de $12,20 \%$. La porosidad superficial ha sido calculada mediante un programa de análisis de imágenes realizadas a las micrografias obtenidas mediante microscopía electrónica de barrido (Fig. 4. a-i), siendo un $2,5 \%$ superior para los sinterizados en atmósfera de nitrógeno.

La Figura 5 muestra la porosidad característica de los aceros sinterizados a $1250^{\circ} \mathrm{C}$ y madurados a distintas temperaturas. Aunque en cada una de las temperaturas se puede notar un amplio intervalo de geometrías y tamaños de poro, se observa un incremento en el porcentaje de porosidad al aumentar la temperatura de maduración de las muestras, disminuyendo al superar los $400^{\circ} \mathrm{C}$. Esto es debido a que al alcanzar la temperatura de maduración de $400^{\circ} \mathrm{C}$ comienza la precipitacion de los nitruros complejos

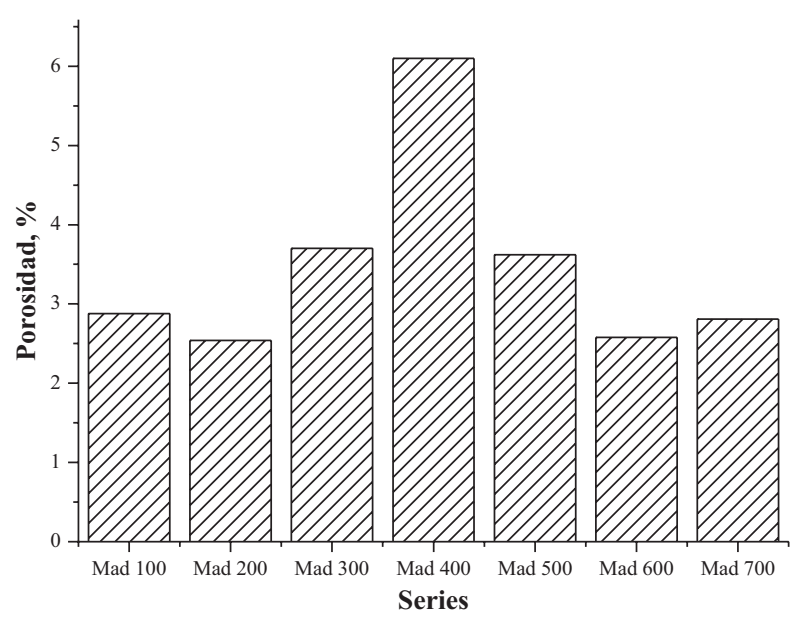

Figura 5. Porcentaje de porosidad en las series maduradas a diferentes temperaturas. 


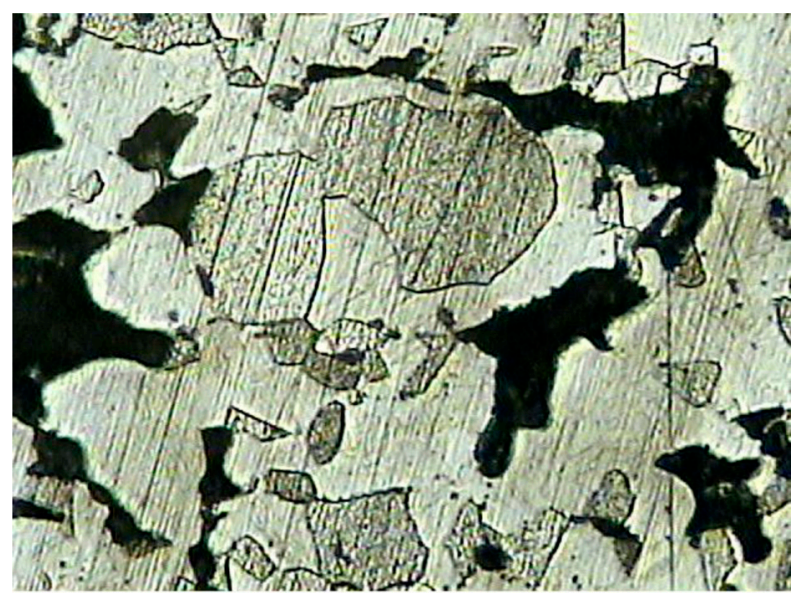

FIGURA 6. Micrografía del acero AISI 430L sinterizado en atmósfera de N-H y templado.

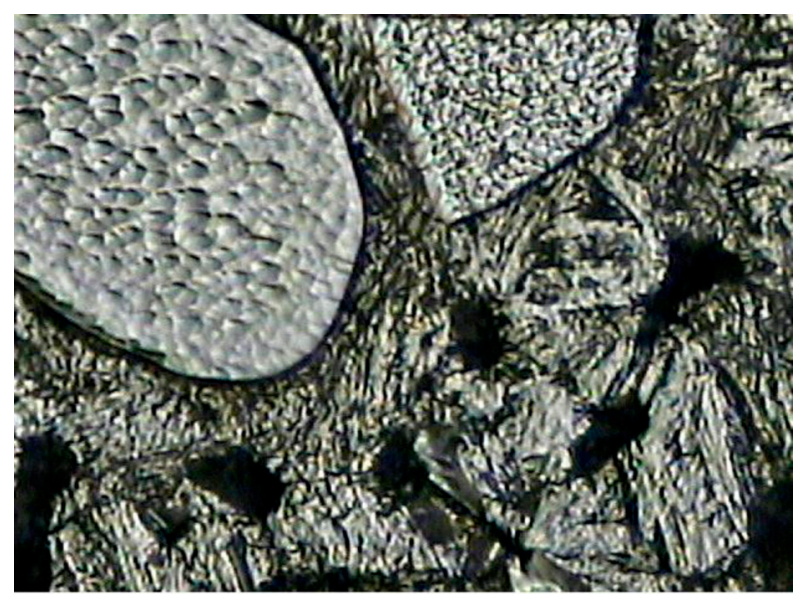

FIGURA 7. Micrografía del acero AISI 430L sinterizado en atmosfera de $\mathrm{N}-\mathrm{H}$ y madurado a $400{ }^{\circ} \mathrm{C}$.

de hierro y cromo en los limites de grano, haciendo la estructura mas compacta y por consiguiente reduciendo la porosidad, como podemos ver en las micrografias de las Figuras 6 y 7 .

Al analizar las propiedades físicas y mecánicas del acero AISI 430L sinterizado en atmósfera de $95 \% \mathrm{~N}_{2}-5 \% \mathrm{H}_{2}$ y de vacío, como son variación dimensional, resistencia a tracción, alargamiento y dureza, se obtienen los datos reflejados en la Tabla 1.

Estos datos confirman que la presencia de los nitruros formados, al sinterizar en atmósfera de nitrógeno, provoca un aumento en las propiedades mecánicas, frente al mismo sinterizado en vacío, junto con una sensible disminución del alargamiento a rotura y de la variación dimensional del acero sinterizado. Esta es la causa de la elección de esta atmósfera para la sinterización de este tipo de aceros, aunque previsiblemente, provocará un efecto perjudicial sobre la resistencia a corrosión de los mismos y sobre la plasticidad. Para solventar esta dificultad, tras la sinterización se procede a la realización de un tratamiento de solubilización, hipertemple y maduración. Para ello se han preparado siete series más a 100, 200, 300, 400, 500, 600 y $700{ }^{\circ} \mathrm{C}$.

\subsection{Dureza}

La dureza HV10 proporciona una primera aproximación del comportamiento mecánico de los materiales, como podemos ver en la Figura 8 de forma que se observa un aumento progresivo de la misma con la temperatura de maduración, debido a la mayor presencia de fase precipitada efectiva en cuanto al mecanismo de endurecimiento se refiere. Cuando la maduración se realiza a temperaturas superiores a los $600^{\circ} \mathrm{C}$, la dureza disminuye drásticamente, debido a que la microestructura resultante corresponde a la misma del acero simplemente sinterizado, como podemos ver en la micrografía de la Figura 9.

\subsection{Resistencia a la tracción}

El ensayo de resistencia a la tracción da información sobre la resistencia del material y sobre su capacidad de alargamiento antes de llegar a la rotura. En la Figura 8 se muestra la mejora de la resistencia a tracción que soportan estos aceros al aumentar la temperatura de maduración, observándose de nuevo una disminución al superar los $600^{\circ} \mathrm{C}$.

La mayor variación, en comparación con los sinterizados en vacío, se observa para los madurados a $600{ }^{\circ} \mathrm{C}$, que llega a ser de un $35,18 \%$.

El límite elástico de estos aceros, como se puede ver en la Figura 9, aumenta suavemente con el aumento de la temperatura de maduración. No se observan grandes diferencias entre las series ensayadas.

Los alargamientos que sufren estos aceros al romperse a tracción se muestran en la Figura 10. Las variaciones son muy pequeñas, observándose valores ligeramente superiores para las temperaturas de 400 y $500^{\circ} \mathrm{C}$. Como se puede apreciar el valor

TABLA 1. Propiedades físicas y mecánicas del acero AISI 430L sinterizado a $1250{ }^{\circ} \mathrm{C}$ en atmósfera de vacío y $\mathrm{N}_{2}-\mathrm{H}_{2}$

\begin{tabular}{lcccc}
\hline SERIE & VARIACION DIMENSIONAL (\%) & RESISTENCIA A TRACCIÓN (MPa) & ALARGAMIENTO (\%) & DUREZA (HV) \\
\hline Vacío & $-2,9$ & 445 & 10,3 & 103 \\
$\mathrm{~N}_{2}-\mathrm{H}_{2}$ & $-1,4$ & 508 & 1,0 & 242 \\
\hline
\end{tabular}




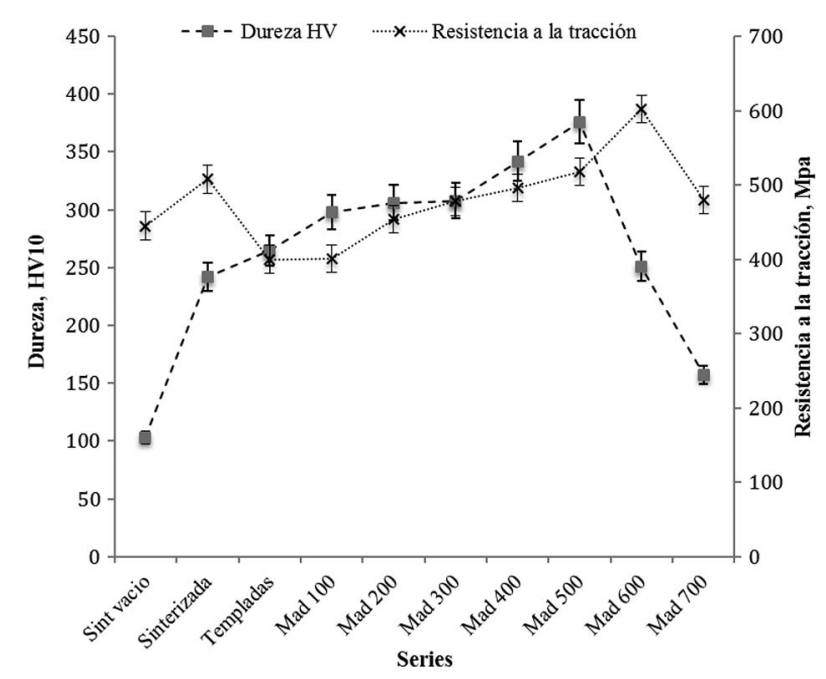

Figura 8. Dureza HV10 y resistencia a tracción de las distintas series de acero inoxidable AISI 430L.

de los sinterizados en vacío es sensiblemente mayor, lo que se traducirá en una menor fragilidad de los mismos.

\section{CONCLUSIONES}

Los valores obtenidos para la porosidad, nos muestran un incremento de la misma para las series sinterizadas en presencia de nitrógeno. Para las series maduradas a diferentes temperaturas, observamos un incremento en el porcentaje de porosidad al aumentar la temperatura de tratamiento térmico disminuyendo al superar los $400{ }^{\circ} \mathrm{C}$, temperatura a la que los compuestos formados por los nitruros y carburos precipitan de forma laminar, pero de tamaño reducido y no formando eutéctico.

La tendencia lógica es un incremento en la resistencia mecánica relacionada con la temperatura de

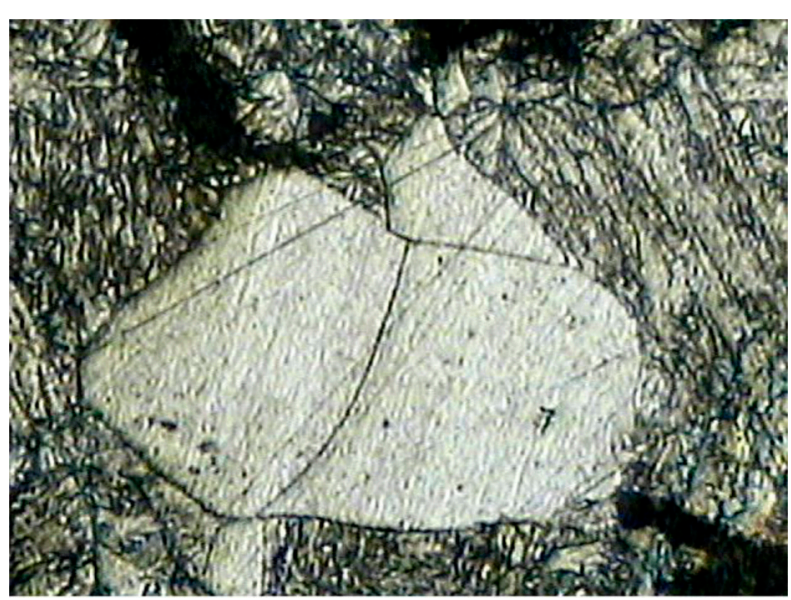

Figura 9. Micrografía del acero AISI 430L sinterizado en atmósfera de N-H y madurado a $700{ }^{\circ} \mathrm{C}$.

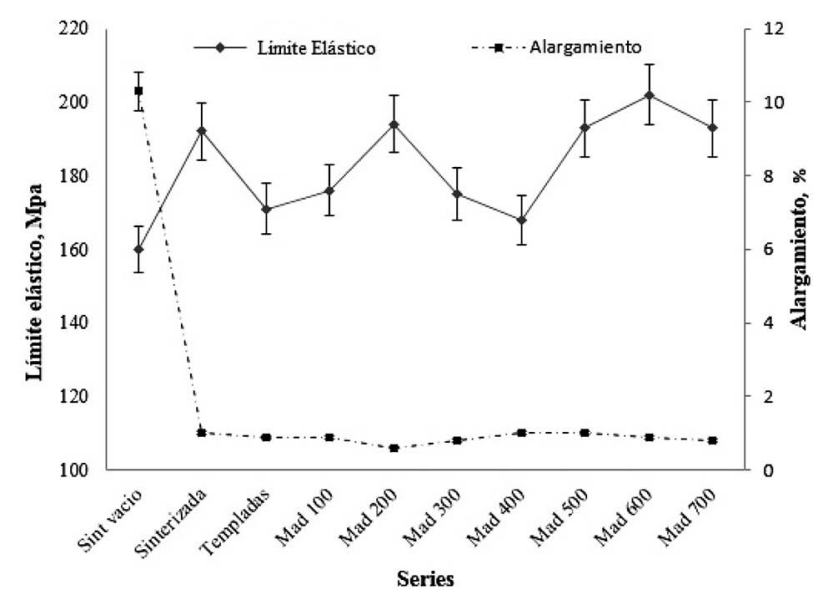

FIGURA 10. Límite elástico y alargamiento de las distintas series.

maduración, debido a la mayor presencia de fase precipitada efectiva en cuanto al mecanismo de endurecimiento se refiere. Cuando la maduración se aproxima a los $700{ }^{\circ} \mathrm{C}$ la microestructura resultante corresponde a la misma del acero simplemente sinterizado; los precipitados, de nuevo con forma laminar, dejan de ser coherentes cristalográficamente con la matriz, lo que se corresponde con unas resitencias mecánicas similares.

\section{REFERENCIAS}

Capus, J.M. 2000. Stainless steel at the 2000 powder metallurgy conference. Adv. Mater. Process. 158, 57-60.

Bautista, A., González-Centeno, A., Blanco, G., Guzmán. S. 2008. Application of EIS to the study of corrosion behaviour of sintered ferritic stainless steels before and after high-temperature exposure. Mater. Charact. 59 (1), 32-39. http://dx.doi.org/10.1016/j.matchar.2006.10.008.

Blanco, L., Campos, M., Torralba, J.M., Klint. D. 2005. Quantitative evaluation of porosity effects in sintered and heat treated high performance steels. Powder Metal. 48 (4), 315322. http://dx.doi.org/10.1179/174329005X82199.

Corpas, F.A., Ruiz-Román, J.M., Codina, S., Iglesias, F.J. 2005. The effect of nitrogen in sintered atmosphere of the ferritic stainless steels AISI 4301 P/M. Rev. Metal. 41, 83-89.

Dobrzanski, L.A., Britan, Z., Grande, M.A., Rosso, M., Pallavicini, E.J. 2005. Properties of vacuum sintered duplex stainless steels. J. Mater. Process. Technol. 162-163, 286292. http://dx.doi.org/10.1016/j.jmatprotec.2005.02.038.

Dobrzanski, L.A., Britan, Z., Grande, M.A., Rosso, M. 2007. Corrosion resistance of sintered duplex stainless steels in the salt fog spray test. J. Mater. Process. Technol. 192-193, 443448. http://dx.doi.org/10.1016/j.jmatprotec.2007.04.077.

Durdaller, C. 1997. Furnace atmospheres. Höganäs Iron Powder Information Hub. 25-26.

Endo, H. 2002. Technology trends in sintered structural parts, Hitachi Podwdered Met. Tech. Rep. Chiba 1, 3-8.

García, C., Martín, F., Blanco, Y., de Tiedra. M. P., Aparicio. M.L. 2009. Corrosion behaviour of duplex stainless steels sintered in nitrogen. Corros. Sci. 51 (1), 76-86. http://dx.doi. org/10.1016/j.corsci.2008.10.003.

García, C., Martín, F., Blanco, Y., Aparicio, M.L. 2010. Effect of ageing heat treatments on the microstructure and intergranular corrosion of powder metallurgy duplex stainless steels. Corros. Sci. 52 (11), 3725-3737. http://dx.doi. org/10.1016/j.corsci.2010.07.023.

Mariappan, R., Kumaran, S., Srinivasa Rao, T. 2009. Effect of sintering atmosphere on structure and properties of 
austeno-ferritic stainless steels. Mater. Sci. Eng. A. 517 (1-2), 328-333. http://dx.doi.org/10.1016/j.msea.2009. 04.011 .

Martin, F., García, C., Blanco, Y. 2011. Effect of chemical composition and sintering conditions on the mechanical properties of sintered duplex stainless steels. Mater. Sci. Eng. A. 528 (29-30), 8500-8511. http://dx.doi.org/10.1016/j. msea.2011.08.013.

Molins, C.J., Bas, J.A., Planas. J. 1992. Stainless Steel: Types and Their Characteristics and Applications. Adv. Powder Metal. Part. Mater. 5, 385-397.

Múnez, C.J., Utrilla, M.V., Ureña, A. 2008. Effect of temperature on sintered austeno-ferritic stainless steel microstructure.
J. Alloy Compd. 463 (1-2), 552-558. http://dx.doi.org/ 10.1016/j.jallcom.2007.09.107

Samal, P.K., Terrel, J.B. 2001. Mechanical properties improvement of PM 400 series stainless steels via nickel addition. Met. Powder Rep. 56 (12), 28-34. http://dx.doi.org/10.1016/ S0026-0657(01)80712-8

Schaefer, D., Trombino, C. 2005. State of the North American $\mathrm{P} / \mathrm{M}$ industry. Adv. Powder Metal. Part. Mater. 1, 1-12.

Sathirachinda, N., Pettersson, R., Wessman, S., Pan, J. 2010. Study of nobility of chromium nitrides in isothermally aged duplex stainless steels by using SKPFM and SEM/EDS. Corros. Sci. 52 (1), 179-186. http://dx.doi. org/10.1016/j.corsci.2009.08.057. 